\title{
In Vitro Visualization of Corneal Wound Healing in an Organ Culture Model Using Multiphoton Autofluorescence and Second Harmonic Generation Microscopy
}

\author{
Wen Lo ${ }^{1}$, Yuh-Ling Chang ${ }^{1}$, Yen Sun ${ }^{1}$, Sung-Jan Lin ${ }^{2}$, Shiou-Hwa Jee ${ }^{2}$, Hsin-Yuan Tan ${ }^{3,4 \#,}$ \\ Chen-Yuan Dong ${ }^{*}$ \\ ${ }^{1}$ Department of Physics, National Taiwan University, Taipei 106, Taiwan \\ ${ }^{2}$ Department of Dermatology, College of Medicine, National Taiwan University and National \\ Taiwan University Hospital, Taipei 100, Taiwan \\ ${ }^{3}$ Institute of Medical Engineering, College of Medicine and College of Engineering, \\ Taipei 100, Taiwan \\ ${ }^{4}$ Department of Ophthalmology, College of Medicine, Chang Gung University and Chang Gung \\ Memorial Hospital, Linko 333, Taiwan
}

\begin{abstract}
The aim of this work is to image the wound healing process of cornea in an in vitro organ culture model with noninvasive multiphoton imaging modality. Autofluorescence and second harmonic generation (SHG) were respectively used to monitor the alterations of cellular and collagenous components during wound healing processes. Within additional developments, this approach may be applied to in vivo visualization of corneal structural destruction and the subsequent regeneration.
\end{abstract}

Keywords: multiphoton autofluorescence, second harmonic generation, cornea, wound healing

Address correspondences to: ${ }^{\#} \mathrm{H} . \mathrm{Y}$. Tan at $\underline{\mathrm{d} 93548010 @ \text { ntu.edu.tw and }}$

*C. Y. Dong at cydong@phys.ntu.edu.tw

\section{INTRODUCTION}

The corneal stroma is a dense connective tissue of remarkable regularity. In the most part, it is made up of collagen embedded in a hydrated matrix rich in proteoglycans $(1,2)$. Most of collagen in corneal stroma is in fibrillar form with precise and narrow diameter. The collagen fibrils predominantly form $2 \mathrm{~m}$ thick, flattened collagenous lamellae which orient parallel to the corneal surface. The collageneous lamellae form a highly organized orthogonal ply and the adjacent lamellae are oriented at right angels with the exception of the anterior stroma. The transparency of cornea is highly dependent upon the regular collagen orientation and inter-fibril distance $(3,4)$. The tissue response in the wound healing process may result in the disruption of the regular collagen organization and therefore degrades corneal transparency. A better modulation of post-surgical wound healing mechanisms may improve the clinical outcomes and prevent complications. Therefore, a mechanistic understanding of corneal wound healing is crucial for managing clinical procedures such as refractive surgeries(5). Non-invasive in vivo imaging technique is significant in understanding wound repair and evaluating the extent of corneal damage.

Confocal microscopy has been applied to the in vivo investigation of corneal infection, dystrophy and the wound healing process of various surgical procedures.(5-13) Cellular components such as nerves, pathogens, inflammatory cells and keratocytes can be observed in vivo by confocal microscopy. However, the inability of visualizing the collagen architecture restricts confocal imaging in studying stromal wound healing. Optical coherence tomography (OCT) is

Ophthalmic Technologies XVII, edited by Fabrice Manns, Per G. Soederberg, Arthur Ho, Bruce E. Stuck, Michael Belkin, Proc. of SPIE Vol. 6426, 642617, (2007) · 1605-7422/07/\$18 · doi: 10.1117/12.699709 
another non-invasive imaging technique that can obtain high-resolution images of the anterior segment. OCT functions by detecting the reflected photons from refractive index mismatched interfaces to visualize stromal lamellae and cells.

In this study, multiphoton microscopy is the preferred modality due to its limited invasion and ability to visualize collagen stroma. The non-central symmetric molecular structure enables collagen to be effective in generating second harmonic signal. Moreover, cytoplasmic autofluorescence from multiphoton imaging provide both morphological and spectral characterization of cells in corneal stroma. The nonlinear process of multiphoton excitation reduced the photodamage during observation and also provides the ability of optical section. Using near infrared as the excitation source, multiphoton microscopy enhances imaging penetration depth in biological tissues. The minimal invasion, enhanced penetration depth and the ability to visualize collagen architecture render multiphoton microscopy applicable for in vivo ophthalmic examination.

In this work, bovine corneal-sclera button is cultured in a biocompatible chamber for long- term and repeated multiphoton observation. The simple organ culture model is used to evaluate the effectiveness of multiphoton imaging in studying the mechanism of corneal wound healing.

\section{MATERIALS AND METHODS}

\subsection{Mutiphoton microscope}

The multiphoton microscope (Fig. 1) we used in this study is a homebuilt system based on a commercial upright microscope (Nikon, E800, Japan.) In order to obtain images at both high resolution and large scale features, a motorized translational stage (H101, Prior, UK) is adapted to the upright microscope. A titanium-sapphire pulse laser (Tsunami, Spectra Physics, Mountain View, CA) operating at the repetition rate of $80 \mathrm{MHz}$ and the wavelength of $780 \mathrm{~nm}$ was used for excitation. A water immersion objective (SFluor, WI, 40X, N.A. 0.8, Nikon) is used to focus laser and acquire multiphoton excited autofluorescence (AF) and second harmonic generation (SHG) from the cornea specimens. To eliminate the reflection from sample, the acquired signals first pass through a short-pass dichroic mirror (700dcspruv$3 p$, Chroma Technology, Rockingham, VT). Prior to reaching the detectors, the broadband autofluorescence and SHG are separated by a secondary dichroic mirror ( $435 \mathrm{dcxr}$, Chroma Technology) and filtered respectively by two band-pass filters (AF: E435lp-700sp, SHG: HQ390/20, Chroma Technology). Photon-counting photomultiplier tubes (R7400P, Hamamatsu, Japan) were used to detect the AF and SHG signals.

\subsection{Long term imaging chamber}

To obtain dynamical information of the infection processes, a corneal culture chamber (Fig. 2) was designed for long term and repeated imaging. The chamber is made of biocompatible polycarbonate (LEXAN TM, General Electronic Company, Fairfield, CT). The cornea chamber is designed to directly adapt to an upright microscope. In our design, the bovine corneal-sclera button is placed in the primary chamber which is sealed by a height adjustable cover with an observation window. The observing window is sealed with a $0.17 \mathrm{~mm}$ thick cover glass for obtaining highly resolved mulitphoton images. The secondary chamber adjacent to the primary chamber has a cap with sterile air filter which allows gas exchange. All the parts are conjoined by biocompatible adhesive (461, Loctite Corporation, Hartford, CT).

\subsection{Bovine corneal model}

Intact bovine eyeballs were obtained from a local market. To avoid unwanted infection, the eyes were first disinfected with $10 \%$ povidone iodine then rinsed by sterilized phosphate-buffered saline (PBS). The corneal-sclera buttons are excised and placed with the epithelium faced down in a sterile container containing PBS. The endothelial corneal cavity was then filled with $1 \%$ agarose gel dissolved in PBS at $40{ }^{\circ} \mathrm{C}$. The cornea along with its supporting gel was later inverted and transferred to the long term imaging chamber. Approximate $4 \mathrm{ml}$ of DMEM was added drop-wise to the central cornea until the limbal conjunctiva was covered and with the epithelial surface exposed to the air. The long term imaging chamber was later removed to an incubator with $5 \% \mathrm{CO}_{2}$ at $37^{\circ} \mathrm{C}$. Prior to multiphoton imaging, the cornealsclera buttons are cultured in our imaging chamber for 1 week. During the observation, the cornea chamber is placed in a temperature controlled environment. 


\subsection{Wounding}

Artificial wound was achieved by cutting the cornea button with a surgical blade and the wound is about $1 \mathrm{~cm}$ in length near the central cornea.

\section{RESULTS}

The multiphoton images of normal bovine cornea are shown in Fig. 3. Comparing to the images of cornea with linear wound shown in Fig. 4 and 5, the morphology of cells in autofluorescence image is not very obvious but we still can identify the characterization of keratocytes (Fig. 3c.) The regular orientation of collagen fibers is clearly demonstrated in Fig. 3d.

After introduction of the linear wound by a \# 11 surgical blade, multiphoton images of similar location in the injured cornea were obtained sequentially in time $(0,1,6,9$ days). Fig. 4 is the multiphoton images obtained just after the injury and demonstrated the early stage of corneal wound healing response. The artificial linear wound can be clearly visualized in the multiphoton images. After wounding, activated keratocytes shown in Fig. 4c and 5c can be identified by autofluorescence and dendritic formation. After 9 days healing, there are signs of keratocyte migration to the edge of wound (Fig 5a and 5c). However, there is no significant collagen regeneration shown in the SHG image (Fig. 5b). The slow regeneration may due to the large gap forbidding keratocytes to migrate cross the wound. This defect can be improved by seeding scaffold in the wound or shrinking the wound size by suturing.

\section{CONCLUSIONS}

Corneal wound healing is a complex process that controlled by a variety of intrinsic factors. The healing process involves cellular proliferation, extracellular matrix synthesis and remodeling. Our results show that multiphoton imaging is effective to monitor the cell morphology, migration and structural alternation of collagen stroma. Our long term imaging chamber provides the feasibility of repeatedly observing the same location of cultured bovine cornea specimens. The ability of long term imaging is helpful in the mechanistic investigation of corneal wound healing process. 


\section{FIGURES}

\section{The Setup of Multiphoton Microscope}

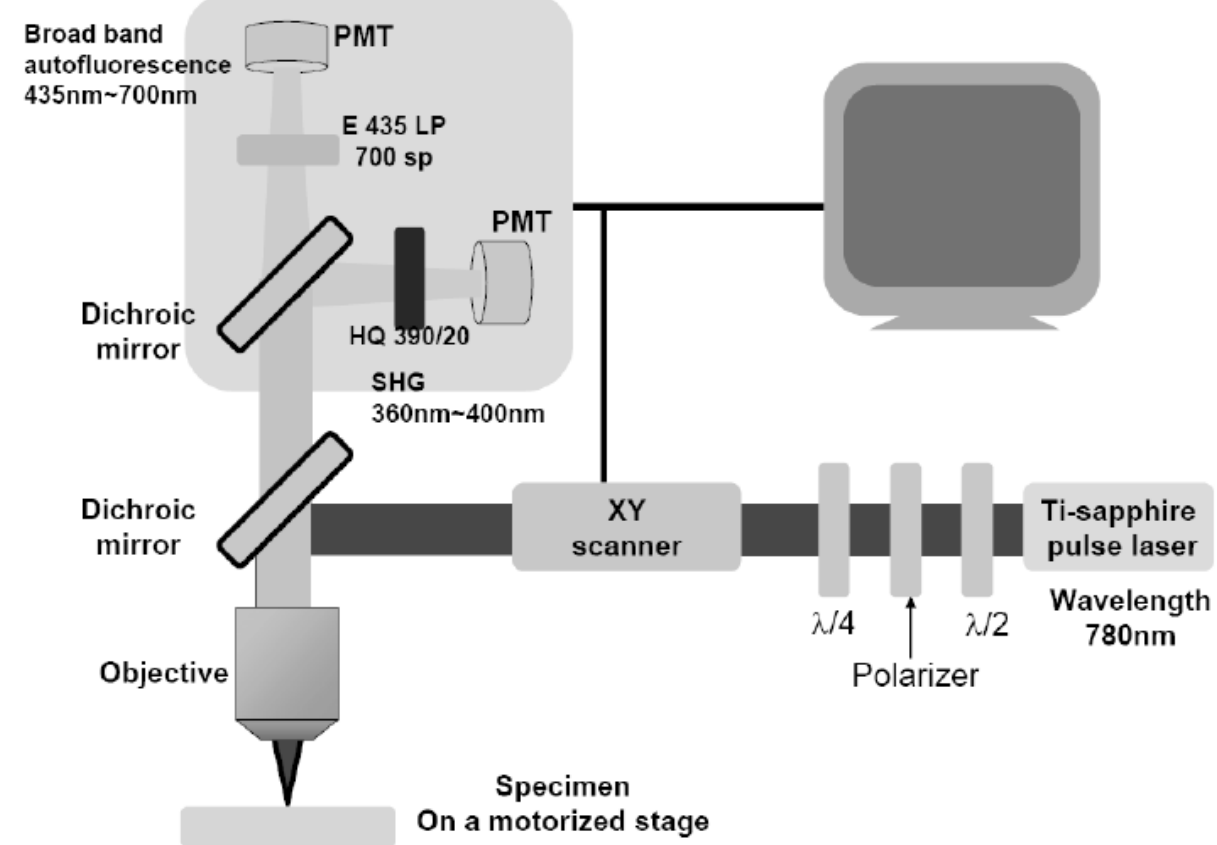

Fig. 1 The schematic setup of our homebuilt multiphoton microscope.
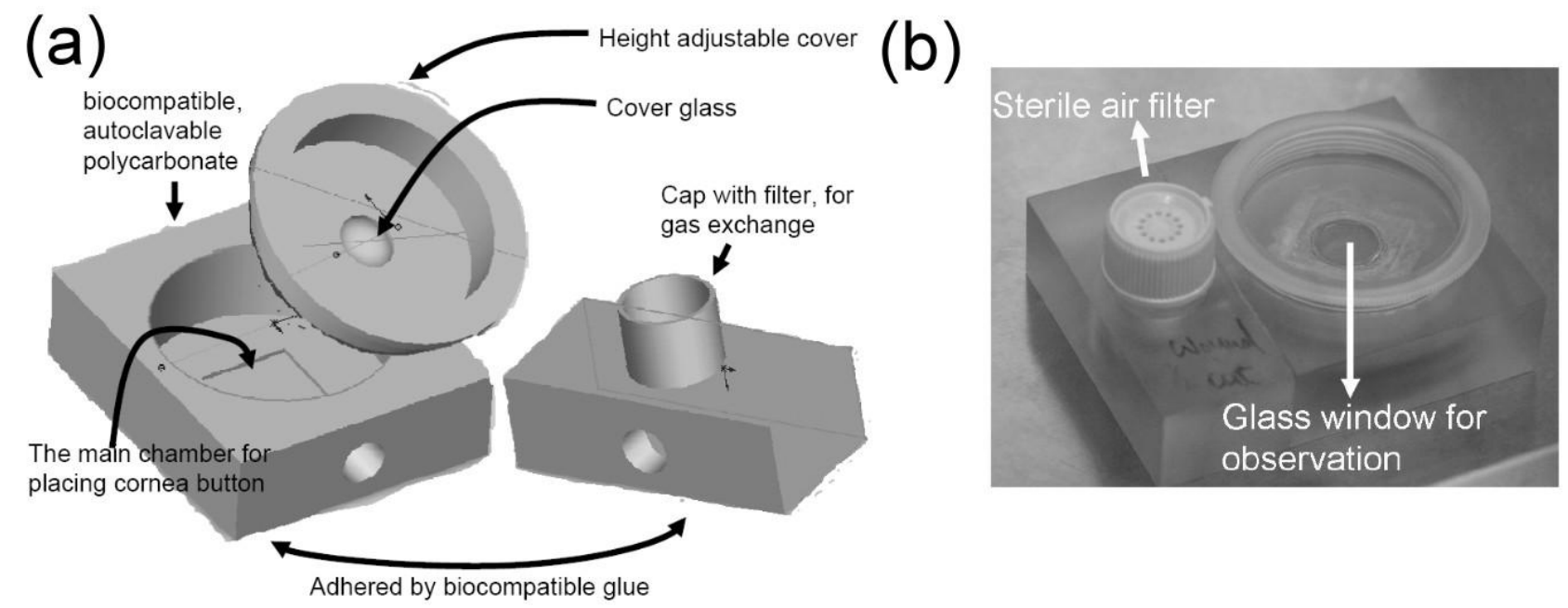

Fig. 2 The in vitro long term imaging chamber made of biocompatible and autoclavable polycarbonate. The chamber can be directly adapted to an upright microscope for high resolution imaging. 

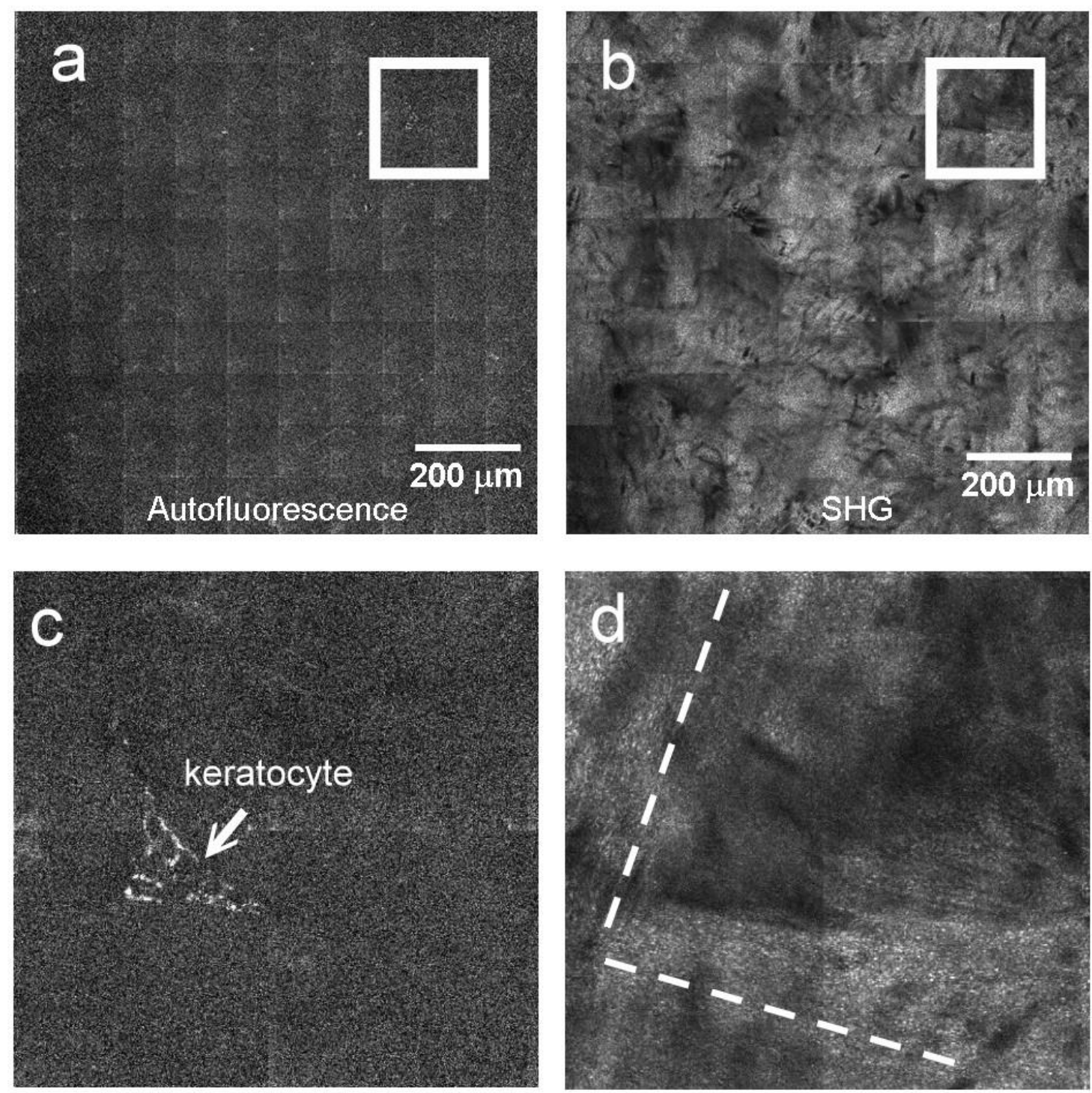

Fig. 3 Multiphoton images of normal bovine cornea cultured in our long term imaging chamber. (a) and (b) are the autofluorescence and SHG images of the normal bovine cornea, respectively. (c) and (d) are the magnified images of the selected area in (a) and (b). 

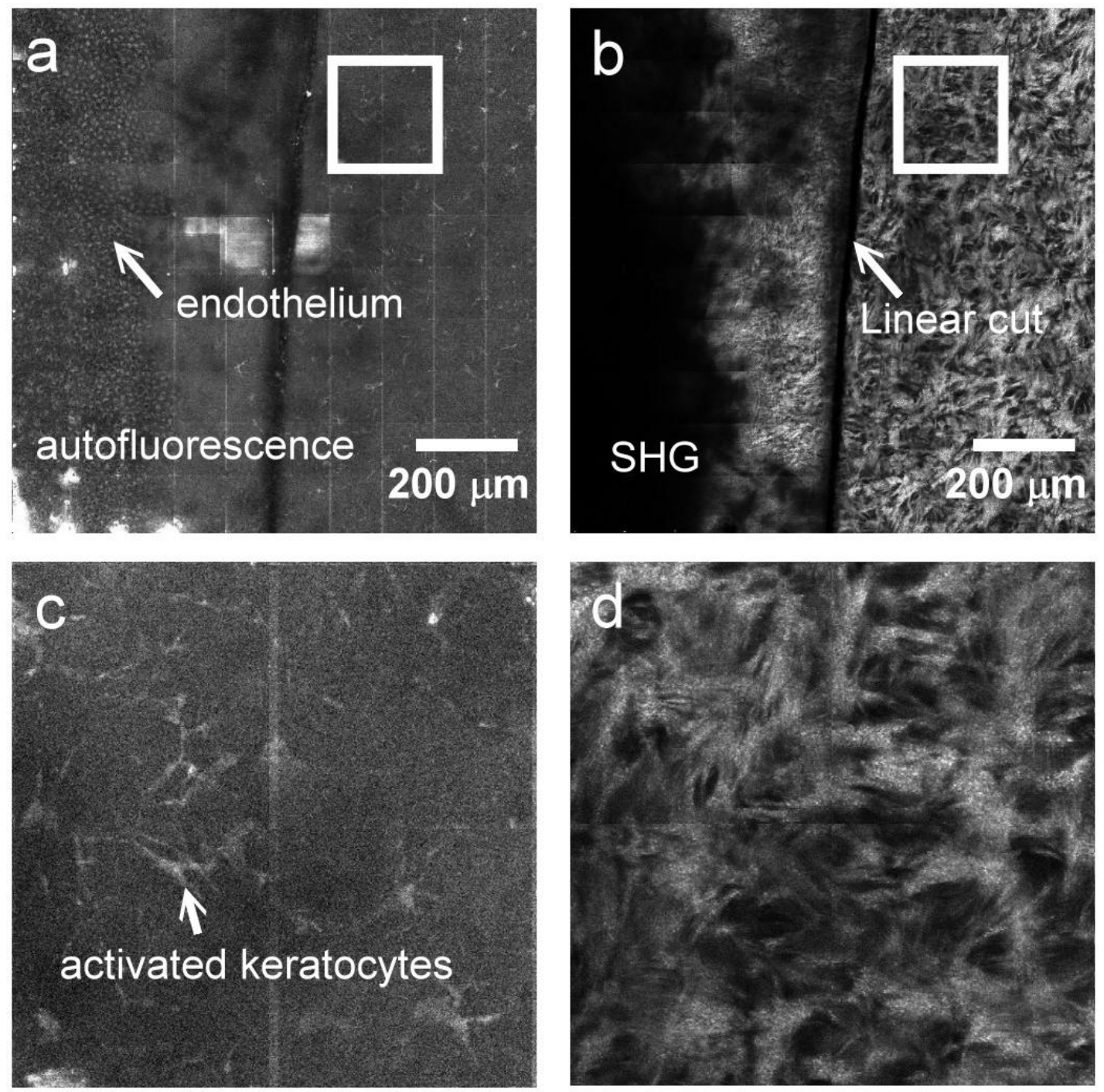

Fig. 2 Multiphoton images of the bovine cornea with a linear cut. These images were obtained at the epithelium/stroma interface just after the wound was made. (c) and (d) are the magnified images of the selected areas in (a) and (b), respectively. 

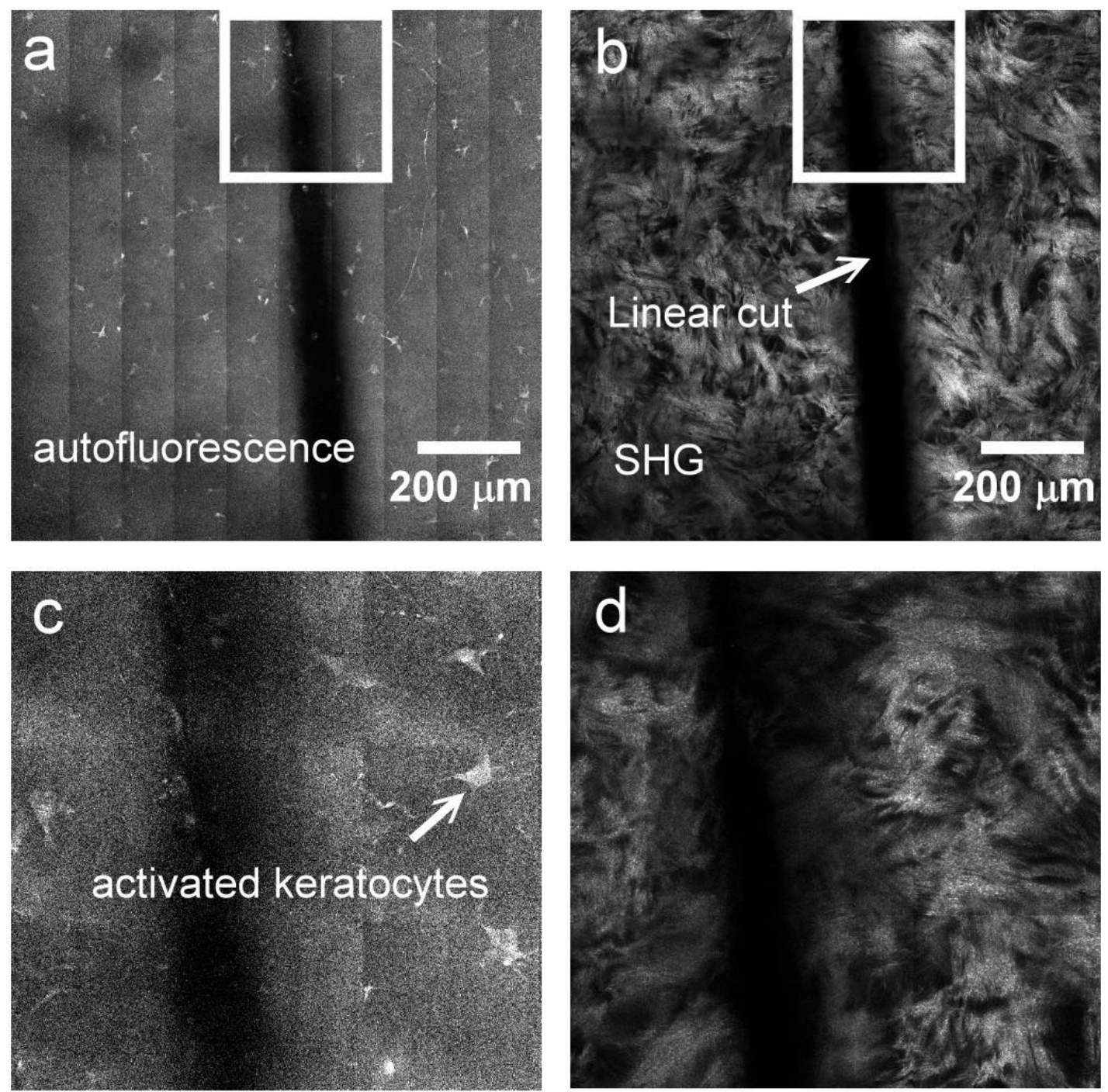

Fig. 3 Multiphoton images of the bovine cornea with 9 days after a linear cut. These images were obtained at $200 \mu \mathrm{m}$ below the epithelium/stroma interface. (c) and (d) are the magnified images of the selected areas in (a) and (b), respectively.

\section{ACKNOWLEDGMENT}

We like to acknowledge the support of the National Science Council, Taiwan and the National Research Program for Genomic Medicine (NRPGM), Taiwan (NSC95-3112-B-002-018). This project was completed using the Optical Molecular Imaging Microscopy Core Facility (A5) of NRPGM. 


\section{REFERENCES}

1. W. W. Y. Kao and C. Y. Liu, "Roles of lumican and keratocan on corneal transparency," Glycoconjugate Journal 19(4-5), 275-285 (2003)

2. H. Tanihara, M. Inatani, T. Koga, T. Yano and A. Kimura, "Proteoglycans in the eye," Cornea 21(7), S62-S69 (2002)

3. D. M. Maurice, "The Structure and Transparency of the Cornea," Journal of Physiology-London 136(2), 263-\& (1957)

4. D. W. Leonard and K. M. Meek, "Refractive indices of the collagen fibrils and extrafibrillar material of the corneal stroma," Biophysical Journal 72(3), 1382-1387 (1997)

5. T. Tervo and J. Moilanen, "In vivo confocal microscopy for evaluation of wound healing following corneal refractive surgery," Progress in Retinal and Eye Research 22(3), 339-358 (2003)

6. A. M. Avunduk, R. W. Beuerman, E. D. Varnell and H. E. Kaufman, "Confocal microscopy of Aspergillus fumigatus keratitis," British Journal of Ophthalmology 87(4), 409-410 (2003)

7. R. W. Beuerman, S. J. Chew, L. Pedroza, M. Assouline, B. Barron, J. Hill and H. E. Kaufman, "Early Diagnosis of Infectious Keratitis with Invivo Real-Time Confocal Microscopy," Investigative Ophthalmology \& Visual Science 33(4), 1234-1234 (1992)

8. H. D. Cavanagh, W. M. Petroll, H. Alizadeh, Y. G. He, J. P. Mcculley and J. V. Jester, "Clinical and Diagnostic Use of in-Vivo Confocal Microscopy in Patients with Corneal Disease," Ophthalmology 100(10), 1444-1454 (1993)

9. G. J. Florakis, G. Moazami, H. Schubert, C. J. Koester and J. D. Auran, "Scanning slit confocal microscopy of fungal keratitis," Archives of Ophthalmology 115(11), 1461-1463 (1997)

10. I. Jalbert, F. Stapleton, E. Papas, D. F. Sweeney and M. Coroneo, "In vivo confocal microscopy of the human cornea," British Journal of Ophthalmology 87(2), 225-236 (2003)

11. D. R. Pfister, J. H. Krachmer, J. D. Cameron, D. J. Doughman and E. J. Holland, "Differentiating squamous cells from melanocytes using confocal microscopy," Investigative Ophthalmology \& Visual Science 37(3), 1618-1618 (1996)

12. K. Winchester, W. D. Mathers and J. E. Sutphin, "Diagnosis of aspergillus keratitis in vivo with confocal microscopy," Cornea 16(1), 27-31 (1997)

13. S. C. Kaufman, R. W. Beuerman and J. Laird, "In-Vivo, Real-Time Confocal Microscopy of Fungal, Bacterial, and Acanthamoeba-Keratitis," Investigative Ophthalmology \& Visual Science 36(4), S1022-S1022 (1995)

14. S. Lin, T. Wu, T. Young, W. Lo, S. Jee and C. Dong, "Quantitative determination of photoaging by use of multiphoton fluorescence and second harmonic generation microscopy," Journal of Investigative Dermatology 125(3), A58-a58 (2005) 